Case report

\title{
Autoimmune thyroiditis - track towards autoimmune polyendocrinopathy type III
}

\author{
Annelie Kérékou Hodé*, Hubert Dédjan \\ Department of Endocrinology, Metabolism and Nutrition, CNHU-HKM Cotonou, Bénin
}

\begin{abstract}
Autoimmune polyendocrinopathies are rare diseases characterized by the coexistence of at least two endocrine diseases linked to an autoimmune mechanism, however sometimes are associated with nonendocrine autoimmune diseases. They are divided into two main subgroups: autoimmune polyendocrinopathy type I and polyendocrinopathies type II-IV. We report a case of a 53-year-old female patient followed for 2 years for Hashimoto's thyroiditis. On admission, she was complaining of polyuropolydipsic syndrome, asthenia, weight loss, abdominal pain and vomiting. The clinical examination noted a dehydrated patient in poor general condition, without fever, tachycardic at 104 beats/min, and polypneic at 24 cycles/min. Laboratory tests revealed hyperglycemia (4.7 g/l), glucosuria, acetonuria, anti-GAD>2000 UI/l antibody, normal TSH. The 8hour cortisol level and anti-21 hydroxylase antibodies level were normal. In this context, the patient was diagnosed with diabetes type 1 associated with Hashimoto's thyroiditis (autoimmune polyendocrinopathy type III). In conclusion, the autoimmune polyendocrinopathy type III is a rare syndrome, predominantly affecting females. In our patient's case, the initial presentation of the disease was dominated by the autoimmune thyroiditis, which is the most frequent endocrine autoimmunity diagnosed in adults with polyglandular autoimmune syndrome. Therefore, the recommended treatment is based on hormonal substitution.
\end{abstract}

Keywords: polyendocrinopathy; autoimmune; type 1 diabetes; Hashimoto's thyroiditis; autoantibodies

\section{Introduction}

Autoimmune

polyendocrinopathies

syndrome (APS) is a rare disease characterized by the coexistence of at least two endocrine diseases linked to an autoimmune mechanism. Still, sometimes there is an association with a non-endocrine autoimmune disease [1-5]. They are recognized as orphan diseases and their coding number is ORPHA282196 [1]. APS is divided into two main subgroups: autoimmune polyendocrinopathy type I juvenile and polyendocrinopathies type II-IV, predominantly

Received: November 2019; Accepted after review: December 2019; Published: December 2019.

*Corresponding author: Annelie Kérékou Hodé, Department of Endocrinology, Metabolism and Nutrition, CNHU-HKM 01 BP386, Cotonou, Bénin.

Tel: 0022921300155

Email: anneliekerekou@yahoo.fr observed in adulthood [1]. The prevalence is evaluated at $1 / 100000$ for APS type I and 1/20000 for APS type II-IV [1]. We report a case of type III autoimmune polyendocrinopathy.

\section{Case report}

We present the case of a 53-year-old female patient followed for Hashimoto's thyroiditis under treatment with Levothyox 100 $\mu \mathrm{g} /$ day for 2 years. Medical history upon admission revealed that she was complaining of polyuropolydipsic syndrome for 3 weeks, asthenia, weight loss, abdominal pain and vomiting. At the clinical examination we observed a dehydrated patient, in poor general condition, apyretic, tachycardic (104 beats/min), with blood pressure at $110 / 60$ $\mathrm{mmHg}$, polypnea at 24 cycles/min, and body 
mass index at $20.5 \mathrm{~kg} / \mathrm{m} 2$. Laboratory tests revealed blood sugar level of $4.7 \mathrm{~g} / \mathrm{l}$, glucosuria, acetonuria , anti-GAD>2000 UI/I antibodies $(\mathrm{N}<2 \mathrm{UI} / \mathrm{I})$, TSH was normal at 2.3 $\mu \mathrm{UI} / \mathrm{I}(0.2-4 \mu \mathrm{UI} / \mathrm{l})$, cortisol levels of $8 \mathrm{~h}$ was normal at $15 \mu \mathrm{g} / \mathrm{dl}(5-18 \mu \mathrm{g} / \mathrm{dl})$, the assay of anti-21 hydroxylase antibodies was negative (normal value $<1 \mathrm{UI} / \mathrm{ml}$ ), hemoglobin at 13.2 $\mathrm{g} / \mathrm{dl}$ and serum calcium at $89 \mathrm{mg} / \mathrm{l}$.

The diagnosis of diabetic ketoacidosis revealing type 1 diabetes associated with Hashimoto's thyroiditis was retained. For financial reasons we didn't perform molecular testing.

Treatment included hydroelectrolytic rehydration and hourly intravenous insulin therapy $(0.1 \mathrm{UI} / \mathrm{kg} / \mathrm{h})$. The clinical outcome was favorable and the treatment at discharge was: lantus 14 units at 19 hours and novorapid 8 units before the three main meals.

\section{Discussions}

Autoimmune polyendocrinopathies type IIIV often manifest between 40 and 60 years of age with a female predominance. The male/female sex ratio is $1 / 3$ [1].

Type III autoimmune polyendocrinopathy codified ORPHA 227982 is defined by the presence of autoimmune thyroid disease and type I diabetes without affecting the adrenal glands. It can be associated with other manifestations such as hypergonadotrophic hypogonadism, autoimmune gastritis, pernicious anemia, celiac disease, chronic inflammatory bowel disease, autoimmune pancreatitis, autoimmunehepatitis, primary biliary cirrhosis, vitiligo, alopecia, urticaria, psoriasis, neurodermitis, rheumatoid arthritis, systemic lupus erythematosus, myasthenia gravis and Sjögren syndrome [1].

Our patient presented the two main criteria for autoimmune polyendocrinopathy type III, with no other clinical manifestations, thus their systematic evaluation was performed at each medical visit. For differential diagnoses we performed laboratory determination of blood count and vitamin B12 to exclude Biermer anemia, measurement of serum calcium and parathormone to exclude hypoparathyroidism, and anti-endomysium antibodies to exclude celiac disease; all results were in the normal reference range.

We were limited in our case by the absence of genetic tests. Given the polymorphism of the involved genes, molecular biology is in fact a domain of research in auto-immune polyendocrinopathies type II-IV.

Several HLA class I and II alleles are mainly implicated in their pathogenesis $[1,6]$. The involvement of other genes has been reported. These are the genes of Protein Tyrosine Phosphatase Non-receptor type 22 (PTPN22), Cytotoxic T-Lymphocyte Associated Antigen (CTLA-4), Vitamin D Receptor, Interleukin 2-Receptor, tumornecrosis-factor- $\alpha$ (TNF- $\alpha$ ), fork-head box P3 (FOXP3), major histocompatibility class I-chain related gene $A$ (MICA), variable number tandem repeats (VNTR) $[1,6]$. These genetic factors alone are not enough to induce these autoimmune polyendocrinopathies because it has been found that despite this genetic susceptibility that there was no hundredpercent concordance in homozygous twins to develop these diseases [1, 7]. Thus, certain environmental factors are also indexed in the triggering of the autoimmune process. Viral and bacterial infections and psychosocial factors have been cited [6-7].

The treatment is based on hormonal substitution of the noted deficits. For patients with monoglandular disease such as Addison's disease, type 1 diabetes or thyroid autoimmune disease, regular testing for other endocrine disorders is recommended because the onset of a second autoimmune disease can vary from one year to twenty years [8]. In our patient this period was two years.

Screening should be done in first-degree relatives and concerns the search for autoantibodies and the monitoring of hormones every 1 to 2 years [8].

\section{Conclusions}

Autoimmune polyendocrinopathy type III remains a rare disease with predominance in female patients. In our patient's case, the initial presentation of the disease was dominated by the autoimmune thyroiditis, 
which is the most frequent endocrine autoimmunity diagnosed in adults with polyglandular autoimmune syndrome. Its treatment is based on hormonal substitution of the proved hormonal deficits. For the screening of first-degree relatives there is no consensus established yet, thus screening is recommended based on the high frequency of autoantibodies observed in these subjects.

\section{References}

1. Frommer L, Kahaly GJ. Autoimmune Polyendocrinopathy. J Clin Endocrinol Metab 2019; 104(10):4769-4782.

2. Betterle C, Dal Pra C, Greggio N, et al. Autoimmunity in isolated Addison's disease and in polyglandular autoimmune diseases type 1,2 and 4. Ann Endocrinol 2001; 62(2):193-201.

3. Betterle C, Dal Pra C, Mantero F, et al. Autoimmune adrenal insufficiency and autoimmune polyendocrine syndromes autoantibodies, autoantigens, and their applicability in diagnosis and disease prediction. Endocr Rev 2002; 23(3):327-364.

\section{Consent}

Written informed consent was obtained from the patient for publication of this case report.

\section{Competing interests}

The authors declare that they have no competing interests.
4. Dittmar M, Kahaly GJ. Polyglandular autoimmune syndromes: immunogenetics and long-term follow-up. J Clin Endocrinol Metab 2003; 88(7):2983-2992.

5. Eisenbarth GS, Gottlieb PA. Autoimmune polyendocrine syndromes. N Engl J Med 2004; 350(20):2068-2079.

6. Kahaly GJ. Polyglandular autoimmune syndromes. Eur J Endocrinol 2009; 161:11-20.

7. Gianni R, Sarvectnick N. Viruses, cytokines, antigens, and autoimmunity. PNAS 1996; 93(6):2257-2259.

8. Kahaly GJ. Polyglandular autoimmune syndrome type II. Press Med 2012; 41(12P2):e663-e670. 